\title{
Sonderausgabe zum Kongress „Pflege 2010“
}

$\checkmark$ Aufgrund der überwältigenden Resonanz auf die erste Sonderausgabe von HeilberufeSCIENCE anlässlich des 7. Gesundheitspflege-Kongresses in Hamburg im letzten Jahr, folgt nun eine zweite Sonderausgabe zum Kongress „Pflege 2010“ am 29. und 30. Januar 2010 in Berlin. Dieser Heilberufe-Kongress gehört angesichts der thematischen Spannbreite und den erfahrungsgemäß mehr als 1.000 Fachbesuchern zu den größten Fortbildungsveranstaltungen in der Pflegebranche.

Die zweite HeilberufeSCIENCE-Sonderausgabe auf www.heilberufe-science.de und www.springerlink.com umfasst die alphabetisch gegliederten Abstracts der meisten Kongressvorträge und -workshops. Diese erstrecken sich inhaltlich über die Themenfelder Pflege-Recht, Pflege-Management und Pflege-Praxis. Diese Sonderausgabe, die frei zugänglich ist, ersetzt die bisher übliche Kongress-CD und erleichtert damit vielen Interessierten aus Forschung und Praxis den dauerhaften Zugang zu den Themen des Kongresses Pflege 2010.

Indem die Kongressabstracts einheitlich in einer zitierfähigen Version vorliegen, steigern sie die Bedeutung der einzelnen Vorträge und Workshops, stärken den TheoriePraxis-Transfer und bieten Anknüpfungspunkte für weitere wissenschaftliche Projekte.
Nun möchten wir Sie einladen, diese HeilberufeSCIENCE-Sonderausgabe kritisch zu lesen und gegebenenfalls auch einen Blick in die anderen Ausgaben von HeilberufeSCIENCE zu werfen. Natürlich würden wir uns ganz besonders freuen, wenn wir Sie auf dem Kongress Pflege 2010 am 29. und 30. Januar 2010 in Berlin begrüßen dürfen.

Ihre

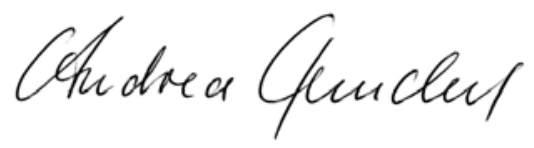

Ihr

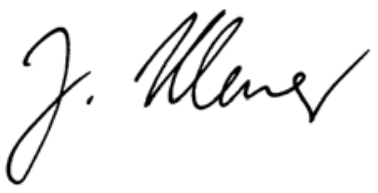

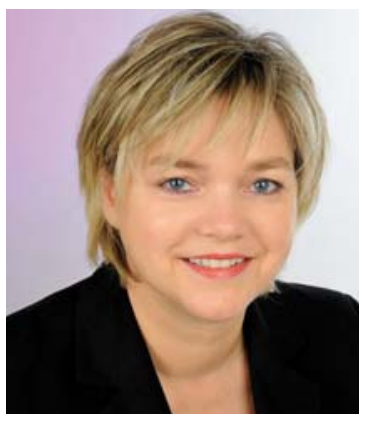

Andrea Tauchert, Berlin

Leitung Kongressbüro andrea.tauchert@springer.com

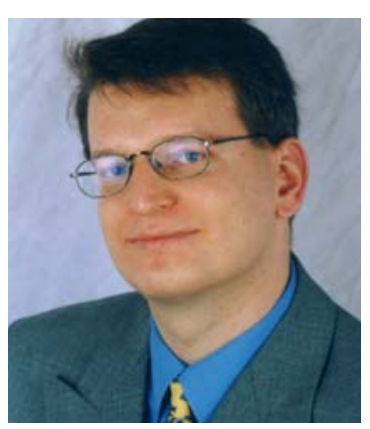

Prof. Dr. med. habil. Jörg Klewer, Zwickau

Schriftleitung heilberufescience@springer.com

HeilberufeSCIENCE 2010; 3 (5): 3 DOI 10.1007/s00058-010-1701-2 